Theaterpraxis zwischen Rhetorilk und Semiotilk: Zur Rolle der Zeichen als Element des Kommunilkationsprozesses

Dr. Nashwa Abou Seada

Abteilung fur Germanistik

Philosophische Fakultät

Menoufia Universität 


\title{
Theaterpraxis zwischen Rhetorik und Semiotik: Zur Rolle der Zeichen als Element des Kommunikationsprozesses
}

\begin{abstract}
Der Bereich der Körpersprache im Rahmen der Theaterpraxis hat sich in den letzten Jahrzehnten im Forschungsbereich zentral etabliert. Der Körper ist in diesem Prozess das Zeichen einer eigenständigen Wirklichkeit. Er fungiert damit in einem System, das Foucault als transparent in dem Sinne bezeichnet hat, dass „der Zeichenträger weitgehend hinter dem Dargestellten, der jeweiligen Bedeutung also,

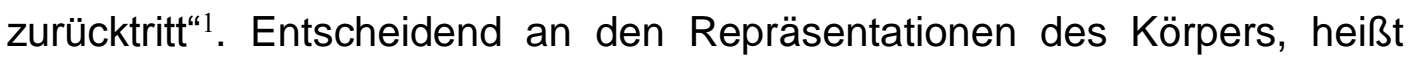
dies, ist nicht ihr "eventuelles Eigengewicht“"2, sondern ihr Verweischarakter, vielmehr sogar ihre Bedeutungsfunktion. Der Körper kann also als Zeichen für den Charakter auftreten. Alles was am Körper beweglich und dadurch veränderbar ist, kann als Zeichen für den Charakter verwendet werden: Mienen, Gesten, Gänge. ${ }^{3}$ Zwischen Körper und „seelischen Modifikationen“4 besteht eine Beziehung. Der Körper und seine Veränderung dienen nämlich als Zeichen bzw. Ausdrucksmittel des Seelenzustands. Inwiefern der Körper als Zeichenträger in der Theaterpraxis und somit im Verhältnis zu Rhetorik und Semiotik steht, ist zentraler Aspekt des Beitrags.
\end{abstract}

1 Galle, Roland: Bilder des Körpers im Roman der Aufklärung. In: Der ganze Mensch: Anthropologie und Literatur im 18. Jahrhundert; DFG-Symposion 1992, hrsg. von HansJürgen Schings. Stuttgart 1994. S. 587.

2 Ebd., S.587.

3 Fischer-Lichte Erika: Semiotik des Theaters. Eine Einführung, Bd.2, 3. Aufl., Tübingen

$$
\begin{array}{r}
\text { 1995, S. } 148 . \\
\text { Ebd., S. } 141 .
\end{array}
$$




\section{لغة الجسد كرمز من رموز الإثارة : الممارسات المسرحية بين البلاغة والسيميائية}

لقد احتل مجال لغة الجسد في إطار الممارسات المسرحية مركزاً مميزاً في البحث العلمي يمنل الجسد في هذا الإطار علامة فارقة من علامات الواقع و بناءًا عليه فيعمل في نظام وصفه فوكو بأنه شفاف بمعنى أن المعنى الحقيقي يكمن حلف هذا الإطارالمرئي وبالتالي يمكن للجسم أن يظهر كعلامة شخصية ويعتبر كل ما هو منقول بواسطة الجسد القابل للتغير علامة من علامات التعبير عن شخصية هناك علاقة بين الجسم و "التعديلات الروحية الجسم وتغييره بمثابة علامة أو تعبير عن حالة الروح "

إلى أي مدى تمثل لغة الإشارة والجسد عنصر أساسي من عناصر ممارسة المسرح، وبالتالي فيما يتعلق بالخطابة والسيميائية هو المحور الرئيسي لهذا البحث يتعرض البحث لعدة قضاياوتساؤلات وهي هل يمكن استخدام كل ما هو متحرك و قابل للتغيير على الجسم منل التعبيرات والإيماءات والممرات كعلامة على الثخصية؟ هل توجد علاقة بين الجسم و "التعديلات العقلية"؟ هل الجسد وتغيره بمثابة علامات أو وسيلة للتعبير عن حالة الروح؟ ما هي أهمية لغة الجسد في إطار الممارسات المسرحية ، وبالتالي فيما يتعلق بالبلاغة وعلم السيميائية؟ البحث يحاول أن يجد اجوبة علمية على هذه التساؤلات . 


\section{Abstract}

\section{The Body Language as a Symbol of Signal: Theatrical Practices Between Rhetoric and Semiotics}

The field of body language is playing a central role in the context of theatrical practices and has become a significant point in scientific research. The body represents an important feature of reality. It works in a system described by Foucault as transparent, meaning that the true meaning lies in this vision. All that is transmitted by the body is subject to change. Morevoer it is a Kind of reflection for the own personality. However there is a deep relationship between the body and the "spiritual alteration body and change it as a sign or expression of the state of the soul"

To what extent the body language is an essential element of the Theater practice and therefore with regard to rhetoric and semiotic is the main aim of this research an the subject of several questions: Is it possible to use everything that is moving and changeable on the body such as expressions, gestures and paths as a sign of personality? Is there a relationship between the body and "mental adjustments"? Does the body and its transformation serve as signs or means of expressing the state of the soul? What is the importance of body language in the context of theatrical practices, and thus with regard to rhetoric and semiotics? The research attempts to find scientific answers to these questions. 


\section{Forschungsstand}

Die Forschung hat ihre Gewichte sehr ungleich verteilt und damit populären Fehlurteilen den Boden bereitet. Sie hat sich auf den Bereich Semiotik konzentriert, während weite Teile des Rhetorischen Bezugs in Hinblick auf die Theaterpraxis Sache weniger Spezialisten geblieben ist. Nur in einigen Untersuchungen, die sich mit verschiedenen Aspekten der Rhetorik beschäftigten, hat dieser Bezug einen Eingang gefunden.

Interessant ist in diesem Kontext die Einführung von Erika Fischer in Theaterwissenschaft. Eine Einführung in die Grundlagen des Faches aus 2009. In der Einführung geht es darum, dass die Theaterwissenschaft nicht als textuelles Verfahren $\mathrm{zu}$ verstehen ist, sondern als sinnliche Wahrnehmung von körperlichen Gesten von Zuschauern und Darstellern. Fischer verteidigt die Aufführungsanalyse als grundlegende Kompetenz. Jede Forschung, die sich nicht auf die eigene körperliche Erfahrung des Theaterereignisses beruft, sollte ach Fischer als Historiographie verstanden werden. Eine Reihe von Aufsätzen die die Semiotik der Theaterwissenschaft ohne jegliche Untersuchung zu den rhetorischen Abhandlungen zeigen sich in einigen Einzelbeiträge wieder. Interessant ist der Beitrag von Gabriele Brandstetter Figura: Körper und Szene zur Theorie der Darstellung im 18. Jahrhundert. Die Wahrnehmung des Körpers und dessen Funktion sowie eine Behandlung der Körpersprache ist Ausgangspunkt, was zu einem besseren Verständnis des Zeichensystem führt.

Ein erwähnenswerter Beitrag der vielmehr die Rhetorik in den Mittelpunkt stellt, ist die Studie von Alexander Košenina. Košenina betrachtet die Körpersprache als wichtiges Ausdruckssystem das er mit dem Wortsystem gleich stellt. Er bezieht sich auf die historische Seite der Rhetorik (Actio-Lehre) sowie auf die Verhaltenslehre (Kunst der 
Verstellung und Dechiffrierung). Besonders wichtig sind seine Ausführungen über die Wechselwirkung zwischen Seele und Körper. Košenina plädiert dafür, dass gerade in der Rhetorik und dem Bereich der Ausdruckspsychologie - von Mienen und Gebärden - der Zusammenhang zwischen der dichterischen Gestaltung und der Theateraufführung liegt.

Für den Forschungsbereich der nonverbalen Kommunikation der eng mit dem Bereich der semiotischen Disziplinen verknüpft ist, beschränkt sich auf den theoretische methodischen Diskurs ohne dass die Semiotik dazu gehört.

Eine Studie, die sich ausschließlich mit dem Zusammenhang von Theaterpraxis zwischen Rhetorik und Semiotik ausführlich beschäftigt, liegt nicht vor. Gleiches gilt für den Einfluss der Rhetorik auf die Theaterpraxis auch hier liegt wenig Forschungsliteratur zu Verfügung.

\section{Theaterpraxis zwischen Rhetorik und Semiotik}

Die Verständigung der Kulturen ist derzeit unter den weltweiten Ereignissen ein sehr aktuelles Thema innerhalb der geisteswissenschaftlichen Forschung. Die Verständigung selber kann aber durch unterschiedliche Wege stattfinden. Es kann entweder über das Wort (verbale Kommunikation) oder auch über die Sprache unseres Körpers (nonverbale Kommunikation) erfolgen. Ohne etwas zu sprechen, treten wir durch unsere Gesichtsausdrücke (Mimik), unsere Körperhaltung (Gestik) mit den anderen in Kommunikation ein. Oft ist es der Fall, dass unser Körper mehr über die innere Haltung verrät als das Wort. Diese Kraft der nonverbale Kommunikation ist Ausgangspunkt des Beitrags.

Diese kurze Einführung sollte die Ausgangshypothese für diesen Beitrag bekräftigen und hebt die Rolle der Körpersprache bzw. der nonverbalen 
Kommunikation als Vermittler zwischen den unterschiedlichen Denkweisen hervor. Ziel ist es, eine Verknüpfung von Actio, auf die Einübung körperlicher Praktiken, den Blick auf die Rhetorik als Körperbildungsmacht im wörtlichen Sinne zu ermöglichen. Eine Ausarbeitung dieser These erfolgt in Bezug auf den anthropologischen Ansatz und soll zeigen, dass sich eine Verbindung von Schauspielkunst, Rhetorik und Sinn etabliert.

Hinsichtlich dieses Hintergrunds wird im ersten Teil des Beitrags auf die Verbindung von Semiotik, Rhetorik und Schauspielkunst näher eingegangen, um im Anschluss im zweiten Teil die Rolle des Zeichen als Element des Kommunikationsprozesses zu erhellen und im letzten Teil die Theatralische Figur des Harlekins exemplarisch heranzuziehen, um dies zu belegen.

\section{Die Verbindung von Semiotik, Rhetorik und Schauspielkunst}

Semiotik und Rhetorik sind miteinander verträglich, da sie sich Kultur und Kommunikation als einen Untersuchungsgegenstand wählen können. Auch die historiographische Aufarbeitung zeigte eine enge Verwandtschaft dieser Disziplinen. Ein Theaterstück als ein Zeichenkomplex aufzufassen, der sich in semiotische Ebenen zergliedern lässt, birgt auch den Richtungsstreit zwischen der Zergliederung in einzelne Zeichen und der Wahrnehmung des Kunstwerks als eines einzigen Zeichens. Egal ob die Schauspielkunst als Zeichenkomplex interpretiert wird oder nicht, es etabliert sich eine Verbindung von Schauspielkunst, Rhetorik und Sinn. Diese schließt sich Umberto Ecos Zeichen-Definition an, die das Zeichen als komplexe Bedeutungseinheit auffasst, die oftmals in Zeichen, jedoch nicht in Figuren geteilt werden können. Für die rhetorische Sichtweise ist es daher sinnvoll, die Kunstwerke nicht als Kunststücke, sondern als Gesellschaft darstellende, kommentierende oder gar verändernde Produkte 
zu definieren, denen sich überzeugende Zeichenfunktionen zuordnen lassen. Es geht also bei der Rhetorik um Operationen, Maßnahmen und Strukturen, die auf kommunikative Effektivität im Sinne einer ganz bestimmten kommunikativen Zielsetzung ausgerichtet sind. ${ }^{5}$

Die Rhetorische Kommunikation ist auf Wechselerzeugung ausgelegt und kann über nonverbalen und performativen Weg überzeugen. Dieser kulturrevolutionäre Aspekt der Rhetorik trifft dabei besonders auf Bereiche zu, die sich vorerst als unkonventionell darstellen. Die Rhetorik ist dabei der entscheidende "Dynamikfaktor" der Kommunikation. Diese Definition von Rhetorik deutet den potenziellen Funktionswechsel von Zeichen nicht nur an, sondern sieht inn als ein Kernelement der rhetorischen Intention.

\section{Die Rolle des Zeichens als Element des Kommunikationsprozesses}

Der Schauspieler erzeugt Zeichen, mit welchen er Bedeutungen bewirkt. Der Zuschauer ordnet diese Zeichen zu und erzeugt Bedeutungen. Nach Rüegg nennt sich dieser Prozess „theatralischer Code."6 Als Folge dessen soll der Schauspieler die Fähigkeit haben, verschiedene Zeichen, Empfindungen und Zustände zu übermitteln. Der Körper und seine Ausdrucksformen werden in diesem Prozess als Zeichen vernommen. ${ }^{7}$ Diese Veränderung der Zeichentheorie hatte einen großen Einfluss auf das Theater und die in ihr enthaltene Psychologie, die ein enges Verhältnis zur

5 Joachim Knape: Rhetorik. In: Klaus Sachs-Hombach (Hrsg.): Bildwissenschaft. Disziplinen, Themen, Methoden. Frankfurt am Main 2005, S.134 ff.

6 Rüegg, Regula: Im Abgehen ein Schnippchen schlagend: Zur Funktion von Kinegrammen in Volksstücken des 19. und 20. Jahrhunderts, Bern u.a. 1991, S. 57f. 7 Vgl. Brandstetter, Gabriele: Figura: Körper und Szene zur Theorie der Darstellung im 18. Jahrhundert. In: Theater im Kulturwandel des 18. Jahrhunderts: Inszenierung und Wahrnehmung von Körper - Musik - Sprache, hrsg. von Fischer-Lichte, Erika. Göttingen 1999, S. 4. 
Schauspielkunst hat. ${ }^{8}$ Die Wendung zum Körper, zu den Sinnen, zum Triebleben, zu den unteren Seelenkräften, rückt die Anthropologie auf die Seite des „Anderen der Vernunft."9 Die Verbindung von Anthropologie und Literatur ist schon lange her. Die Literatur selbst ist über weite Teile anthropologisch ${ }^{10}$. Sie ist ein Schauplatz für anthropologische Themen und Motive geworden. Während die Anthropologie sich mit dem ganzen Menschen befasst, so will auch die Literatur den Menschen ganzheitlich in seiner Körper-Seele-Einheit erfassen, eine „Physiognomische Verknüpfung von Inneren und Äußeren."11

In der zweiten Hälfte des 18. Jahrhunderts wird die Anthropologie zur „Lehre vom Menschen“ gekrönt und verbindet somit die Seelenlehre mit der Physiologie. ${ }^{12}$ Die Hochschätzung der Körpersprache mit gewissen kulturund geistesgeschichtlichen Strömungen treten zum ersten Mal auf. ${ }^{13}$ Während der Aufklärungszeit wurden diese gegen 1750 ergriffen und bezweifelt. Erst mit Hugo von Hofmannsthal ${ }^{14}$ wurde um 1902 die Bezeichnungskraft der Worte ${ }^{15}$ vorgelegt. Die Schätzung bzw. Renaissance der dramatischen Gebärdensprache, der Pantomime, die über das Theater hinaus eine bedeutende Rolle hat, zeichnete sich also erst um 1900 aus. ${ }^{16}$

So basiert beispielsweise die italienische Commedia dell'arte auf Stereotypen, die in der Sächsischen Typenkomödie, und selbst im

$8 \quad$ Vgl., Košenina, Alexander: Anthropologie und Schauspielkunst. Studien zur eloquentia corporis' im 18. Jahrhundert, Tübingen 1995, S. 8 f.

9 Ebd. S. 5.

10 Pfotenhauer, Helmut: Literarische Anthropologie; Einführung. In: Ebd., S. 557.

11 Ebd., S. 555.

12 Košenina, S. 9.

13 Ebd., S. 290.

14 Gemeint hiermit sind Hofmannstahls Äußerungen in dem bekannten Brief an Lord Chandos indem er die Sprache als unfähig bezeichnet da sie es nicht schafft ins "Innere der Dinge zu dringen." Vgl. Hofmannsthal, Hugo von: Ein Brief. In: Ausgewählte Werke in zwei Bänden, hrsg. von Rudolf Hirsch. Stuttgart 1960, S.462.

15 Košenina weist auch darauf hin, dass vor Hofmannstahl erste Überlegungen existierten wie etwa von Ekhof oder Garrick. Siehe hierzu Košenina, S.291.

16 Košenina, S. 290 
Bürgerlichen Trauerspiel des 18. Jahrhunderts wiederkehren. In der modernen Epik haben sich Typen entwickelt, wie etwa die Schelme (Picaro) in Grimmelshausens Simplicius Simplicissimus (1668/69), Thomas Manns Felix Krull (1957). Interessant sind bestimmte Typen die hinaus Handlungen, wie zum Beispiel das Initiationsmodell des Bildungsromans. ${ }^{17}$

Die Autoren versuchen, ihre erworbenen Menschenkenntnisse in poetische umzusetzen. ${ }^{18}$ Das spiegelt sich am meisten in der Zeichenlehre, und den „Anleitungen zu physiognomischen Verknüpfungen“19, die man anhand der Gestaltungsformen der Figuren wieder erkennen konnte. So etwa in den Körperzeichen von Figuren wie dem Harlekin bzw. der komischen Figur. Mit Hilfe dieser Figur konnte die Schauspielkunst bzw. die Literatur „anthropologische Denkformen in literarische Gestaltungsformen“20 verwandeln. Die Körpersprache gewinnt hier die Kernbedeutung und ist ein Vermittler von authentischen Gefühlen:21
„Tränen, beredte Gesten, stumme Blicke, warme Händedrücke zeigen zugleich das Versagen der sprachlichen Kommunikation und ihre Steigerung als Körpersprache. Es ist die Sprache des Gefühls, die Sprache der Aufrichtigkeit, die nun über den Körper ihre Authentizität ausdrückt.“22

Der Körper ist in diesem Prozess das Zeichen einer eigenständigen Wirklichkeit. Er fungiert damit in einem System das Foucault als transparent

17 Frauke Berndt: Poetische Topik. In: Zymner, Rüdiger. Handbuch Literarische Rhetorik. Berlin: 2015. De Gruyter, S. 435.

18 Košenina, S. 15.

19 Pfotenhauer, Helmut: Literarische Anthropologie; Einführung. In: Ebd., S. 557.

20 Ebd. S. 557.

21 Trummeter, Birgit: Die Ohnmacht - Inszenierungen eines Phänomens von Körperlichkeit in der französischen Literatur des 18. Jahrhunderts, Diss., Uni Mannheim 1999, S. 15.

22 Schwanitz, Dietrich: Der Körper zwischen Rhetorik und Symptomatologie. In: Kapp, Volker: Die Sprache der Zeichen und Bilder, Marburg 1990, S. 125. 
in dem Sinne bezeichnet hat, dass „der Zeichenträger weitgehend hinter dem Dargestellten, der jeweiligen Bedeutung also, zurücktritt." ${ }^{23}$ Entscheidend an den Repräsentationen des Körpers, heißt dies, ist nicht ihr „eventuelles Eigengewicht“ ${ }^{24}$, sondern ihr Verweischarakter, vielmehr sogar ihre Bedeutungsfunktion.

Der Körper kann als Zeichen für den Charakter auftreten. Alles was am Körper beweglich und dadurch veränderbar ist, kann als Zeichen für den Charakter verwendet werden: Mienen, Gesten, Gänge. ${ }^{25}$ Zwischen Körper und „seelischen Modifikationen"26 besteht also eine Beziehung. Der Körper und seine Veränderung dienen nämlich als Zeichen bzw. Ausdrucksmittel des Seelenzustands. ${ }^{27}$ Aufbauend darauf sollte der Schauspieler:

„[...] diejenigen Veränderungen des Körpers oder seiner Teile in Ansehen ihrer Lage und Figur hervorbringen, welche mit gewissen Veränderungen in der Seele harmonisch sein können." 28

So gesehen sind die verschiedenen Gesten ein Ausdrucksmittel der Seele. Am meisten zeigt sich das in der Theaterpraxis anhand von der Harlekin-Figur. In der Figur des Harlekins erhöht sich der Gehalt von Mimik und Gestik erheblich. Eine breite Skala von mimischen Reaktionen zeigen sich. Es lässt sich als aussagekräftiges Mittel des wahren bzw. natürlichen, besser gesagt des authentischen Ausdrucks von Empfindungen verstehen.

23 Galle, Roland: Bilder des Körpers im Roman der Aufklärung. In: Der ganze Mensch: Anthropologie und Literatur im 18. Jahrhundert; DFG-Symposion 1992, hrsg. von Hans-Jürgen Schings. Stuttgart 1994. S. 587.

$24 \quad$ Ebd., S.587.

25 Fischer-Lichte Erika: Semiotik des Theaters. Eine Einführung, Bd.2, 3. Aufl., Tübingen 1995, S. 148.

26 Ebd., S. 141.

27 Ebd., S. 142.

28 Ebd., S. 143. 
Der Einsatz von Gebärde als Ausdruck von Gedanken oder Empfindungen ist mit einer direkten Wiedergabe der "Stimme des Herzens“29 zu vergleichen. Das Herz steht in diesem Kontext für Reinheit bzw. Echtheit und Natürlichkeit dieser Gebärden, die zu rein sind um gefälschte Empfindungen vorzutäuschen. Auf dieser Basis formt und funktionalisiert sich der Harlekin auf der Bühne. Dieser handelt genau so rein, spielerisch und natürlich wie ein Kind. Er springt auf der Bühne, ferner versucht er die Bedeutung der Zeichensprache auszusprechen, indem komplette Szenen in pantomimische bzw. mimischen Szenen voll mit Gestik überfüllt sind.

Durch „Gebärden“ werden aber nicht nur Empfindungen geäußert, vielmehr werden auch mittels Körperzeichen und Gebärden alle Fragestellungen beantwortet. Die Theaterpraxis spiegelt eine „ungekünstelte Gestikulation“30, die sich auch in einem hohen Anteil auf die Gestik beschränkt. Die körperliche Beredsamkeit wirkt hier als Ersatz zur gesprochenen Sprache. Anhand der Funktionalisierung des Harlekins zeigt sich, inwiefern er die von der Frühaufklärung, in Verbindung mit der zunehmenden Aufwertung von Emotionalität und Sinnlichkeit, Spielraum für die Thematisierung des Körpers erschloss. Die „Figurationen des Körpers"31, wie sie nun anhand des Harlekins zur Sprache kommen, haben ihre Grundlage in der Sprache des Körpers. Diese gilt nicht nur dem Körper selbst, sondern einer Realität. Eine große Aufmerksamkeit gilt hier der Rolle des Körpers als Zeichen. Ein Zeugnis für die wichtige Rolle, die diese Zeichenfunktion und die damit verbundene Körpersprache einnimmt.

29 Zitiert nach Košenina, S. 22. Košenina stützt sich hier auf die Begriffsbestimmung der „Gebehrden“ von J. G. Sulzer: Allgemeine Theorie der schönen Künste II von 1775.

30 Hinck, 1965, S. 243

$31 \quad$ Roland, Galle, S. 588. 
Der Körper wird somit zum Zeichenträger gemacht, dessen „eigene Expressivität hinter seiner Verweisungsfunktion“32 stecken. Darüber hinaus versteckt sich der Effekt des „Bezauberers“ der Sinne zu, was eine Aufwertung der Sinnlichkeit mit sich bringt. Diese Aufwertung fand im 18. Jahrhundert nur auf der Ebene der „medialen Vermittlung“33 statt, wobei sie durch die körperliche Abwesenheit bedingt war. ${ }^{34}$ Mit der Wendung gegen die Barocktradition erfolgte der Durchbruch der Affektenlehre und nachfolgend der Anthropologie. Affekte und Mienen werden als Wirkungen der Natur verstanden. „Actio“ wird den natürlichen Gaben zugerechnet und die körperliche Beredsamkeit enthält ästhetischen Eigenwert neben der Wortsprache. ${ }^{35}$ Von Besonderer Bedeutung ist die Betonung der körperlichen Anwesenheit auf der Bühne in Verbindung mit der medialen Vermittlung durch die körperliche Beredsamkeit, die dem Harlekin zugesprochen ist. Der Körper wird daraufhin zu einem zentralen Medium der Selbstpräsentation und des Kommunikationsprozess.

\section{Die Figur des Harlekins als exemplarisches Beispiel}

Der Hanswurst oder Harlekin ist die Figur, die diese Zeichen am besten übermitteln konnte, da sich in der komischen Figur alles vereint, was der normativen Poetik widerstand: Sinnlichkeit, Illusionsdurchbrechung, Unwahrscheinlichkeit, Normfreiheit und Handlungszwecke, die über das Spiel hinausgehen. ${ }^{36}$

32 Ebd., 588.

33 Koschork Albrecht: Alphabetisation und Empfindsamkeit. In: Der ganze Mensch:

Anthropologie und Literatur im 18. Jahrhundert; Hrsg. Von Hans Jürgen Schings.

Stuttgart/Weimar 1994, S.628

34 Ebd., S. 628

35 Gees, Marion: Schauspiel auf Papier: Gebärde und Maskierung in der Prosa Robert Walsers. Berlin 2001, S.23 ff.

36 Fulda, 2005, S. 361. 
„Endlich kann man Körper und Seele in ihren gegenseitigen Verhältnissen, Einschränkungen und Beziehungen zusammen betrachten, und das ist es, was sich Anthropologie nennt“ ${ }^{\text {*37 }}$

Die Anthropologie fungiert hier als wichtiges Verbindungselement, mit dem Schauspielkunst zwischen den zwei Disziplinen - der psychischen und physischen - zusammen kommt. Die Bühne ist somit der Ort der „physischen Menschendarstellung“38. Schaut man sich die Figur des Harlekins in Bezug auf diese These an, so erkennt man, dass er verschiedene Gesten anführt, die er als ein Ausdrucksmittel der Seele verwendet. Durch den Harlekin wird der Gehalt von Mimik und Gestik erhöht. Eine breite Skala von mimischen Reaktionen zeigt sich durch das Erstaunen, die Überraschung, die Verblüffung und das Schrecken, die unterschiedliche Empfindungen ausdrücken. Dieses Aufkommen der Mimik in der Schauspielkunst - wie es Johann Jakob Engel in seiner Schrift Ideen zu einer Mimik ausführlich dargestellt hat - lässt die Personen als Individuen hervorstechen, was einen Wandel in der Schauspielkunst erzeugt. Wichtig ist es, die richtige Verwendung der Zeichensprache zu beachten, da es eine der wichtigsten Aufgaben der Schauspielkunst ist, vor allem da sie je nach Kontext unterschiedlich verstanden werden können.

Es finden sich komplette pantomimische bzw. mimische Szenen voll mit Gestik überfüllt. Diese Funktionalisierung des Harlekins zeigt, inwiefern die zunehmende Aufwertung von Emotionalität und Sinnlichkeit, Spielraum für die Thematisierung des Körpers erschließt. Exemplarisch ist die Figur des Harlekins der als Zeichenträger fungiert, dessen „eigene Expressivität

37 Platner, Ernst: Anthropologie für Aerzte und Weltweise, 1772, zitiert nach Hans Jürgen Schings, Vorbemerkung. In: Der ganze Mensch: Anthropologie und Literatur im 18. Jahrhundert; hrsg. von Hans Jürgen Schings. Stuttgart/Weimar 1994, S. 5.

$38 \quad$ Schletterer, Daniela Weis: Das Laster des Lachens: Ein Beitrag zur Genese der Ernsthaftigkeit im deutschen Bürgertum des 18. Jahrhunderts. Wien 2005, S. 78. 
hinter seiner Verweisungsfunktion"39 stecken. Die Harlekin-Figur spielt in diesem Kontext wohl die wichtigste Rolle, die eine besondere Körpersprache schafft. Die Signale, die durch die Körpersprache gegeben werden, gleichen die gesprochene Sprache aus. Mehr körperlicher Ausdruck auf der Bühne ist für also wichtig. ${ }^{40}$ Der Körper wird daraufhin zu einem zentralen Medium der Selbstpräsentation und der Symbolisierung sozialer Zugehörigkeiten. ${ }^{41}$ Die Harlekin-Figur bzw. die komische Figur ist durch ihre Regeldurchbrechung sehr geeignet für eine wechselseitige Selbstrepräsentation und sozialer Zugehörigkeit. ${ }^{42}$ So etwa die Gestaltung von unteren sozialen Schicht auf der Bühne. Diese Figur versucht auf der Bühne durch eine Reihe von körperlichen Zeichen wie etwa Gesten und mimischen Ausdrucksmitteln die Unterdrückung dieser sozialen Schicht dem Zuschauer zu vermitteln. Der Harlekin spricht somit derbe Wahrheiten bzw. Realitäten aus, die mittels der gesprochenen Sprache schwer auszudrücken sind. ${ }^{43}$. Der Harlekin dient also nicht nur der Belustigung, vielmehr wirkt er als verweisende Figur sowohl auf der sozialen Ebene als auch auf der Erziehungsebene.

Durch der neuen Verweisfunktion des Harlekins wird der anthropologische Bedarf an „Mündigkeit, Subjektsein und Selbstdenken.“

40 Vgl. Brandstetter, Gabriele: Figura: Körper und Szene zur Theorie der Darstellung im 18. Jahrhundert. In: Theater im Kulturwandel des 18. Jahrhunderts: Inszenierung und Wahrnehmung von Körper - Musik - Sprache, hrsg. von Fischer-Lichte, Erika. Göttingen 1999, S.29.

41 Meuser, Michael: Zwischen „Leibvergessenheit“ und „Körperboom. "Die Soziologie und der Körper Between, "Body Oblivion" and "Body Boom": Sociology and the Body, Sport und Gesellschaft - Sport and Society, Jahrgang 1, Heft 3. Stuttgart 2004, S. 197-218. Vgl. S. 202.

42 Fischer, Lichte Erika: Semiotik des Theaters, 1995, S. 54. Fischer schreibt hierzu, dass die gestischen Zeichen als Repräsentation des Ichs sehr geeignet sind. Da der Harlekin als Figur sich kaum an die Regeln hält, ist er als Charakter sehr geeignet, um die durch seine verschiedenen gestischen Zeichen verursachte Regelbrechung als Repräsentation eines wechselseitigen Ichs zu deuten.

43 So wie etwa der Narr im 17. Jahrhundert. 
erweckt. ${ }^{44}$ Diese Funktionsmodifikation des Harlekins signalisiert eine Psychologisierung der Figuren. Die Körpersprache spielt also die Rolle der Ersatzfunktion, mit deren Mittel das ausgedrückt wird, was man mit der gesprochen Sprache nicht ausdrücken kann bzw. will: Das Seufzen, die gesenkten Augen und die Atemlosigkeit auf der Bühne gelte als Paradebeispiel einer Selbstpräsentation und geben der Situation Rührung und einen viel stärkeren Ausdruck als die Sprache allein.

\section{Ergebnisse der Forschung}

Abschließend ist hier festzuhalten, dass sich ein langsamer Anstieg der Wertschätzung der körperlichen Beredsamkeit des Harlekins zum Erziehungs- und Belehrungszweck zeigt. Die Dominanz der mimischen Ausdrucksweise in der Theaterpraxis beweist körperliche Beredsamkeit, verkörpert in der Figur des Harlekins und seiner Tendenz zur „Wirklichkeitserfassung." 45 Die Figur des Harlekins verwirklicht hier die Idee der Verbindung des Rhetorik mit der Semiotik in der Theaterpraxis, indem er durch die Körpersprache das "ganze psycho-physiologische Ereignis, das dem Werden eines Wortes vorangeht" 46 , realisiert.

Das neu Konzipieren und der Wandel der Ausdrucksintention der körperlichen Beredsamkeit stellt den Zusammenhang und den Wandel dar, der in der Theaterpraxis und dem in ihr enthaltenen anthropologischem Konzept erzeugt wird. Diese Beherrschung von mimischen komischen Mitteln zugunsten eines scharfen Blicks „für gesellschaftliche Spannungen

44 Pfotenhauer, Helmut: Literarische Anthropologie; Einführung, Der ganze Mensch: Anthropologie und Literatur im 18. Jahrhundert; hrsg. von Hans Jürgen Schings, Stuttgart/Weimar 1994. S. 555.

$45 \quad$ Ausdruck hier übernommen von Hinck, 1965, S. 251.

46 Hofmannsthal, H.v.: in: Reden und Aufsätze. S. 470 f. 
und moralisch satirische Themen der Zeit" ${ }^{47}$ macht meines Erachtens die Verbindung von Theaterpraxis, Rhetorik und Sinn möglich.

Darüber hinaus könnte der Körper an sich als wichtigste Kommunikationsmittel betrachtet werden. Die Zeichen werden in einem gewissen System übernommen. Durch die Übertragung der Zeichen wird eine Sprache vermittelt die mithilfe des Zuschauers bzw. des Empfängers aufgenommen wird ohne eine verbale nach Foucault auch "transparente“ Kommunikation in Betracht zu ziehen. Schließlich kommt man zur Schlussfolgerung, dass der Körper daraufhin zu einem zentralen Medium des Kommunikationsprozess wirkt ohne dessen Rolle die Kommunikation unmöglich ist. 


\section{Literaturverzeichnis}

Brandstetter, Gabriele: Figura: Körper und Szene zur Theorie der Darstellung im 18. Jahrhundert. In: Theater im Kulturwandel des 18. Jahrhunderts: Inszenierung und Wahrnehmung von Körper - Musik - Sprache, hrsg. von Erika Fischer-Lichte. Göttingen 1999.

Fischer-Lichte, Erika: Kurze Geschichte des deutschen Theaters. Tübingen 1993.

Dies.: Semiotik des Theaters: Das System der theatralischen Zeichen. Eine Einführung, Bd.1. Tübingen 1983.

Dies.: Semiotik des Theaters: Vom „künstlichen“ zum natürlichen Zeichen- Theater des Barock und der Aufklärung, Bd. 2. Tübingen 1989.

Dies.: Semiotik des Theaters: Die Aufführung als Text, Bd.3,4. Aufl., Tübingen 2003.

Dies.: (Hrsg.): Theater im Kulturwandel des 18. Jahrhunderts: Inszenierung und Wahrnehmung von Körper - Musik Sprache. Göttingen 1999.

Dies.: Der Körper als Zeichen und als Erfahrung. In: ebd., S. 53-68.

Frauke, Berndt: Poetische Topik. In: Zymner, Rüdiger. Handbuch Literarische Rhetorik. Berlin 2015

Fulda, Daniel: Schau-Spiele des Geldes. Die Komödie und die Entstehung der Marktgesellschaft von Shakespeare bis Lessing. Tübingen 2005. 
Galle, Roland: Bilder des Körpers im Roman der Aufklärung. In: Der ganze Mensch: Anthropologie und Literatur im 18. Jahrhundert, hrsg. von Hans-Jürgen Schings; DFGSymposiom 1992. Stuttgart 1994.

Gees, Marion: Schauspiel auf Papier: Gebärde und Maskierung in der Prosa Robert Walsers. Berlin 2001.

Hinck, Walter: Das deutsche Lustspiel des 17. und 18. Jahrhunderts und die italienische Komödie,Commedia dell'arte und théâtre italien. Stuttgart 1965.

Ders.: Die europäische Aufklärung. Frankfurt am Main 1984.

Hofmannsthal, Hugo von: Ausgewählte Werke, in zwei Bänden, hrsg. von Rudolf Hirsch. Stuttgart 1960.

Ders.: Gesammelte Werke, Reden und Aufsätze II, 1914-1924, Aufzeichnungen 1889-1929, hrsg. von Bernd Scholler und Rudolf Hirsch. Frankfurt am Main 1980.

Koschork, Albrecht: Alphabetisation und Empfindsamkeit. In: Der ganze Mensch: Anthropologie und Literatur im 18. Jahrhundert; Hrsg. von Hans Jürgen Schings. Stuttgart/ Weimar 1994.

Košenina, Alexander: Anthropologie und Schauspielkunst. Studien zur, eloquentia corporis‘ im 18. Jahrhundert. Tübingen 1995.

Meuser, Michael: Zwischen „Leibvergessenheit“ und „Körperboom.“ Die Soziologie und der Körper Between, "Body Oblivion" and "Body Boom": Sociology and the Body, Sport und Gesellschaft - Sport and Society, Jahrgang 1, Heft 3. Stuttgart 2004. 
Pfotenhauer, Helmut: Literarische Anthropologie; Einführung, Der ganze Mensch: Anthropologie und Literatur im 18. Jahrhundert, hrsg. von Hans Jürgen Schings. Stuttgart/ Weimar 1994

Platner, Ernst: Anthropologie für Aerzte und Weltweise, 1772, zitiert nach Hans Jürgen Schings, Vorbemerkung. In: Der ganze Mensch: Anthropologie und Literatur im 18. Jahrhundert; hrsg. von Hans Jürgen Schings. Stuttgart/Weimar 1994.

Rüegg, Regula: Im Abgehen ein Schnippchen schlagend: Zur Funktion von Kinegrammen in Volksstücken des 19. und 20. Jahrhunderts, Bern u.a. 1991.

Schletterer, Daniela Weis: Das Laster des Lachens: Ein Beitrag zur Genese der Ernsthaftigkeit im deutschen Bürgertum des 18. Jahrhunderts. Wien 2005.

Schwanitz, Dietrich: Der Körper zwischen Rhetorik und Symptomatologie. In: Kapp, Volker: Die Sprache der Zeichen und Bilder, Marburg 1990.

Sulzer, J. G. : Allgemeine Theorie der schönen Künste II von 1775.

Trummeter, Birgit: Die Ohnmacht - Inszenierungen eines Phänomens von Körperlichkeit in der französischen Literatur des 18. Jahrhunderts, Diss., Uni Mannheim 1999. 